\title{
A novel method for natural motion mapping as a strategy of game immediacy
}

\author{
Ji Young Lee ${ }^{1}$ and Tack Woo ${ }^{2}$ \\ ${ }^{1}$ Graduate School of Culture Technology, Korea Advanced Institute of Science and Technology \\ Daejeon, Korea \\ [e-mail: dontsigh@kaist.ac.kr] \\ ${ }^{2}$ Department of Digital Contents, Kyung Hee University \\ Gyeonggi-Do, Republic of Korea \\ [e-mail: twoo@khu.ac.kr] \\ *Corresponding author: Tack Woo
}

Received November 13, 2016; revised October 13, 2017; ; revised January 13, 2018; accepted January 2, 2018; published May 31, 2018

\begin{abstract}
The method of operating a game could determine the psychological distance between the player and the game character, and thus, in the Virtual Reality, players' control methodologies are important to enhance their immersion. This study has the objective of examining the difference in games according to the method of operation based on the player's movements. This study researched the effect of the method of operating movement conforming to the movement of the character and the physical operation of the body on forming game experiences for the player. The result of performing an experiment increased reality for the game player through a controller in the shape of the actual control, to increase focus in the game. As so, game play through movements, including actual movements by the player displayed to enhance game satisfaction. In the part of media remediation field, Game can be defined as media which has their own unique hypermediacy. Especially, in the motion based game, players' movement mediates players and the game, therefore, players' movement could make players' experience augmented or immediate in accordance with the characteristics of movements. Even though sports and dances genres of motion-based games are common, RPG or adventure genres are rare. It can be explained that the characteristics of the action have been explained in the immediacy. In a game of fantasy, which is difficult to experience in real-life situations, the nature of the player's motion can increase the immersion of the game, which can contribute to utilization of players' motion and experience design in the various genres and suggestion of grounds theory. In addition, through this study, it is able to design motion-based games of various genres.
\end{abstract}

Keywords: Traffic classification, unsupervised learning, k-nearest neighbor, clustering

A preliminary version of this paper appeared in $2^{\text {nd }}$ EECCS 2016, August 10-13, Qingdao, China. This work (Grants No. S2449657) was supported Project of convergence/integrated technology development funded Korea Small and Medium Business Administration in 2016. 


\section{Introduction}

As video game technologies evolve and game design becomes more sophisticated, players possess more options to achieve higher levels of expression [1]. With a conventional keyboard or joystick to control in-game movement, various interfaces may be applied to the video game. The amount of player control varies according to the interface. Because the innate characteristics of the technology limit in-game interactivities and player interaction, which are embodied in the technology, the possible variations in these experiences are limited [2]. Body motion is the latest interface used in video games. Its main characteristic is true spatial tracking of the user's motion and gestures, allowing users to interact in and control elements of the 3D gaming world with their bodies [2].

Motion-based games are defined as those that use human body motions as interfaces for operating games, but some of them still have tangible game controllers and limit full body interaction with games. For example, musical instruments and driving equipment-such as guitars, drums, steering wheels, and brakes - were the initial means of incorporating human body motions into games. However, such motions were confined to operating realistically shaped game controllers, and players' body posture or location had no significant meaning in the games.

Several studies were conducted showing that body motion affected game players' engagement positively [3], [4], [1]. However, this research was performed in an experimental, control-based game environment, and no research has been done on the relationship between human motions and players' experience. Because in an intangible, motion-based game environment the natural motions are the prominent characteristic, players' gaming performance, such as ease of control, could be improved. Until now, sports and dances have constituted the main content of motion-based games, because it has been convenient, given that those genres use natural motions. Fantasy or shooting games, however, have posed difficulties in terms of using natural motion. Because these genres include many factors that do not exist in daily life, arbitrary motions could symbolize characters' motions, and in these cases, such motions were applied through such instrumentation as game pads, joysticks, and the keyboard or mouse.

The motion-based interface improves the interaction between the game and the player, and at the same time maximizes the player presence compared to the previously utilized game interface. The method remodels a game's mediation. The media's mediating method can be classified into two types: the immediacy strategy, which tries to make the audience or user forget the media's mediation to secure the media's transparency; and the hypermediacy strategy, which draws a clear line between the media and life to emphasize the mediating experience. Game media are developing vis-à-vis the efforts to remodel and transform their mediating method [11]. Thus, it can be said that realistic-computer-graphic- and physics-engine-based detailed gameplay can improve the transparency of game media, and that gameplay devices such as the keyboard, mouse, or joystick have a hyper-mediating characteristic. The mediating method of games, however, is different from the twofold mediating method of a photograph, film, or TV. Games are based on the players' active involvement and therefore have an interactive characteristic. As games, unlike other types of media, are created for high immersion, they usually aim for a media immediacy method, and the game interface is applied to decrease the hypemediacy, maintaining the interactivity between the game and the players, and to try to remove the difference between the real world 
and the game world. From partial bodily motion such as shaking or moving the controller to camera-based full-body-motion gameplay, the motion-based interface has been developing towards non-mediating experience. Researches on understanding games as media usually explain the players' non-mediating experience with various elements, such as presence, flow, and immersion, but there has been insufficient research on the immediacy experience for different types of motion interface. According to a recent research on the partial-bodily-movement method with a controller and the full-body-motion interface in terms of the level of embodiment and player experience [9], the presence and enjoyment improve as the embodiment level increases. The game interface's embodiment level, however, has been categorized based on sensory immersion and motor immersion, and therefore, research has been conducted on motion utilization for players, which they can use for gameplay. As motion itself can function as a new interface, research is necessary for the type rather than level of embodiment, and how the former can affect the player experience. With regard to the game player's experience, gaming experience has so far not been connected to real-life experience [10], and it is meaningful to look into whether a player's gaming experience depending on the embodiment type remodels his/her previous gaming experience. This research trend could be found in the field of image retrieval algprothms [23], which proved users' interpretations about images could narrow dowm the semantic gap.

For our research, we established a set of both natural and arbitrary motions. We examine empirical data concerning motion-based game players' perceived naturalness of motions and the degree of presence, enjoyment and gaming performance. We also present tips for the future development of motion designs.

\section{The definitions of gaming experiences, mental model and motion mapping}

\subsection{Immediacy experience as remediation strategy}

As a game's players can directly control their gaming experience, the user immersion in games is higher than that in other media. The sense of control is the immediacy strategy of games as media, and game players can feel immersion because of this [11]. Motion-based game information removes the controllers mediating between the game and the player, allowing the player to experience game presence, as if he/she really exists in the game world. This maximizes the game medium's immediacy strategy. The hyper-mediating sense of control through motion improves the player's game presence, flow, and identification, and ultimately strengthens the player's non-mediating experience and empathy towards the game.

The motion-based interface re-mediates the controller, remodels the game medium, and enhances the user experience. A game with the previous format stops at making the user experience that of the experience of the game [10] while the user experience with the motion-based interface can bring about identification with the game experience as the actual user experience. In the same way that the smartphone's haptic and tangible interface routinized digital experience as daily activity [12], when the players use the same motions in a game as the actual space, their experience expands from that of simply playing a game to real life, thus remodeling the previous game experience.

\subsection{Presence, Immersion and performance}

Presence essentially refers to the sense of existing or "being there" in a virtual space. The International Society of Presence Research defines presence in computer-mediated 
environments as a "psychological state in which even though part or all of the individual's current experience is generated by and/or filtered through human-made technology, part or all of the individual's perception fails to accurately acknowledge the role of the technology in the experience” [5]. Players' motion interaction with games offers greater presence because camera based motion capture technology allows players to move unconsciously than do video games with just controllers. If players' pose and location are gauged, the feeling of "being there" is higher when players move their bodies naturally, as they would in everyday life.

The concept of immersion is widely used in discussing digital games and gameplay experiences[14]. Immersion can be defined as "the sensation of being surrounded by a completely other reality [...] that take over all of our attention, our whole perceptual apparatus[15]". Even though these terms have vague difference in meaning, as presence was originated from Virtual Reality (VR) studies it is more like physical transport experience.

Because body movement based games have different input-output systems, in the simulated media players would immerse easily and feel presence deeply. Without movements, players just control input devices and watch out their character, so the world is distinguished definitely - players' world in the reality and characters' world in the game. However, in the body movement based games, players' world and game world is unified because players' movements are the same as characters'. Therefore, this paper suggests more specific definition of game experiences in related with immersion and presence.

Brown and Cairns[16] structured three stage of immersion. The first step was about engagement which was consisted of access, controls and feedback. Secondly, engrossment stage came up and visual, task and plot were the key factors. Finally, total immersion was needed empathy and atmosphere. Fundamental factors for immersion were related to game interfaces and ways of play. And then, game contents and context became the second level. Similarlly, Ermi and Maya[14] divided sensory immersion, challenge-based immersion and imaginative immersion. Sensory immersion is the fundamental concept of immersion, and audio-visual aspects of games are involved this concept. Challenged-based immersion is the next phase and imaginative immersion is the ultimate, in this phase players emphasized with their game characters and immersed game fantasy fully.

In order to achieve ultimate immersion, interfaces and gameplay methods are the first step and especially in movement based games, movements are related to physical transportation experience. Different movement patterns influence to the feeling of control and these experiences would affect to players' immersion.

Slater et al.[17] suggested a positive relationship between presence and the degree of body movement of participants. The results revealed a significant positive association in the active group between body movement and presence. However, they did not assert the direction of causality but proposed a two-way relationship. These findings pointed that body movement may enhance sense of presence.

Immersion and presence is consisted of game experiences with slight differences. Tan[18] explained due to the belief that being present in an environment makes a person able to respond emotionally to events and characters contained within the mediated experience, in the researches of entertainment products, many terminologies and definitions were established. However, in the above-mentioned findings, immersion researches estimated from mainly conventional games and presence was measured in the VE(Virtual Environment), not a game content. So in this study, we would suggest that how players feel immersion and presence during playing games and full body movement influence on these experiences. 
Gaming performance is an essential parameter for usability, so for the development of natural motion mapping in the future, it is desirable for a high presence, obtained through natural motion mapping, to contribute to gaming performance. Natural motion mapping enables players to take action easily, so the time for task completions is shorter than for arbitrary motions.

\subsection{Mental Model and motion mapping}

Mental models, which are formed through previous personal and social experiences, can represent both actual and hypothetical events [6]. In terms of motion-based games, players' mental models help them to make decisions about how to move and take poses in gaming situations. For instance, when players face a battle, their motions are affected by mental models that dominate their judgments about how to attack and when to defend. Their natural motions are related closely to their mental models.

Mapping refers to how the actions performed by users of interactive media are connected to corresponding changes in the mediated environment [13]. Natural mapping is defined as how closely actions represented in a game match the actions that would be used to bring about that change in a real environment [7], [8]. Hence, natural motion mapping uses realistic motions from real-life behaviors. For example, if a game character should walk, the player would march in place. To make a character brandish a sword, players would swing their arms. Even though players do not have swords, their mental models provide information about how to use them. In this situation, natural motion mapping is helpful in enacting mental models and narrowing the gap between the real and the hypothetic, giving players the feeling of "being there.”

\section{Experimental setting and model}

\subsection{Experimental setting}

Natural motion mapping and arbitrary motion mapping are mainly about modes of expression in game play. The natural motion mapping was designed to capture realistic motions, such as walking in place or swinging a sword. Arbitrary motion mapping was composed of simple motions that symbolized specific instructions. For example, walking was represented by raising the left arm in a straight forward position, and swinging a sword was expressed by raising the right arm straight forward. Game motion in this experiment were classified 6 different actions, and each 3 actions depend on higher tasks such as navigation and battle (Table 1).

Might and Magic: Dark Messiah was chosen for experiment because this game has a first-person point of view with a human as the protagonist. These features meet the requirement for players' engagement in the full body interaction situations. Participants were not likely to have played it before because it is less popular than other games. Game tasks were redesigned to be navigation and battle centric. For navigation centric tasks, players were forced to find five hidden barrels(Table 2). Battle centric tasks consisted of beating seven enemies with a sword, using the same maps as for the navigation task. The 40 participants all played the game with both natural and arbitrary motion mapping and were afterward surveyed with a questionnaire. We also measured the time taken by each task mode. We anticipated that more familiar motion mapping would decrease the time needed to control the action and would offer higher efficiency. 
Table 1. Examples of game motions

\begin{tabular}{|l|l|l|l|}
\hline \multicolumn{2}{|c|}{} & Natural motion mapping & Arbitrary motion mapping \\
\hline \hline \multirow{2}{*}{$\begin{array}{l}\text { Navigation } \\
\text { motions }\end{array}$} & Walk & Walking in place & Raising left arm horizontally \\
\cline { 2 - 4 } & Jump & Jump & Lifting left leg \\
\cline { 2 - 4 } & Switch & Step left or right & Swing arms left or right \\
\hline \multirow{3}{*}{$\begin{array}{l}\text { Battle } \\
\text { motions }\end{array}$} & Attack & Brandishing right arm & Raising right arm horizontally \\
\cline { 2 - 4 } & $\begin{array}{l}\text { Strong } \\
\text { attack }\end{array}$ & Swatting with folding hands & Raising right arm \\
\cline { 2 - 4 } & Defense & Guarding face with two hands & $\begin{array}{l}\text { Raising right hand and leaning the } \\
\text { head }\end{array}$ \\
\hline
\end{tabular}

Table 2. Experimental setting

\begin{tabular}{|c|c|c|}
\hline & \multicolumn{2}{|c|}{ Mode of motion mapping } \\
\hline \hline $\begin{array}{c}\text { Set of game } \\
\text { tasks }\end{array}$ & $\begin{array}{c}\text { Navigation centric task: Finding } \\
\text { and crashing 5 barrels }\end{array}$ & $\begin{array}{c}\text { Battle centric task: Beating 7 } \\
\text { enemies with a sword }\end{array}$ \\
\hline
\end{tabular}

A game player's motions were mapped for movement and battle, measured in 15 points (Fig. 1). The game players played the game using natural and arbitrary motion . It should be noted that natural motion shows big movements and utilizes various parts of the body while arbitrary motion utilizes the arms and legs, and is only partial motion. All the motions, however, were mapped according to keyboard-based game control, regardless of the motion type. After the gameplay, a survey was conducted to analyze the players' gaming experience, satisfaction, and preferences. The survey questionnaire included questions selected from among the survey questions formulated by Witmer and Singer [19] and Jennett et al. [20]. The gaming performance was investigated based on the time that was spent to complete the "navigation-centric task" and the "battle-centric task."

The arbitraty movents consisted of easy-to-learn actions, with the left arm representing the navagation centric task and the right arm representing the battle centric task. This is because in PC based game play, players let their cahracter move using the keyboard with their left hand and move the mouse with their right hand to perform tasks such as combat. In addition, since it is common for the hand to extend forward when expressing the meaning of straight forward, the straight forward motion is constituted as a reference, and the motions such as backward, leftward and rightward walking are constituted. The most typical configurations of the detailed operation is shown in Table 3.

Natural movements were relatively easy to express, but with the constraint of keeping distance from the screen, the movements like forward can not be configured to actually move forward. Therefore, the forward movement is expressed as a standstill, and the leftward, rightward and backward movements are defined as moving ranges to the corresponding region based on the point at which the forward movement is performed (Fig. 2). 


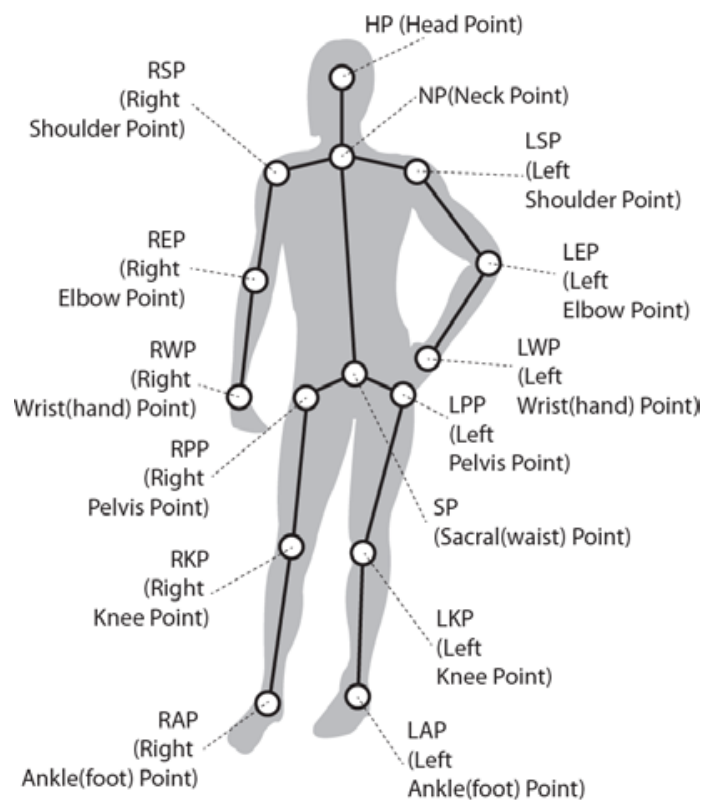

Fig. 1. Motion measurement points

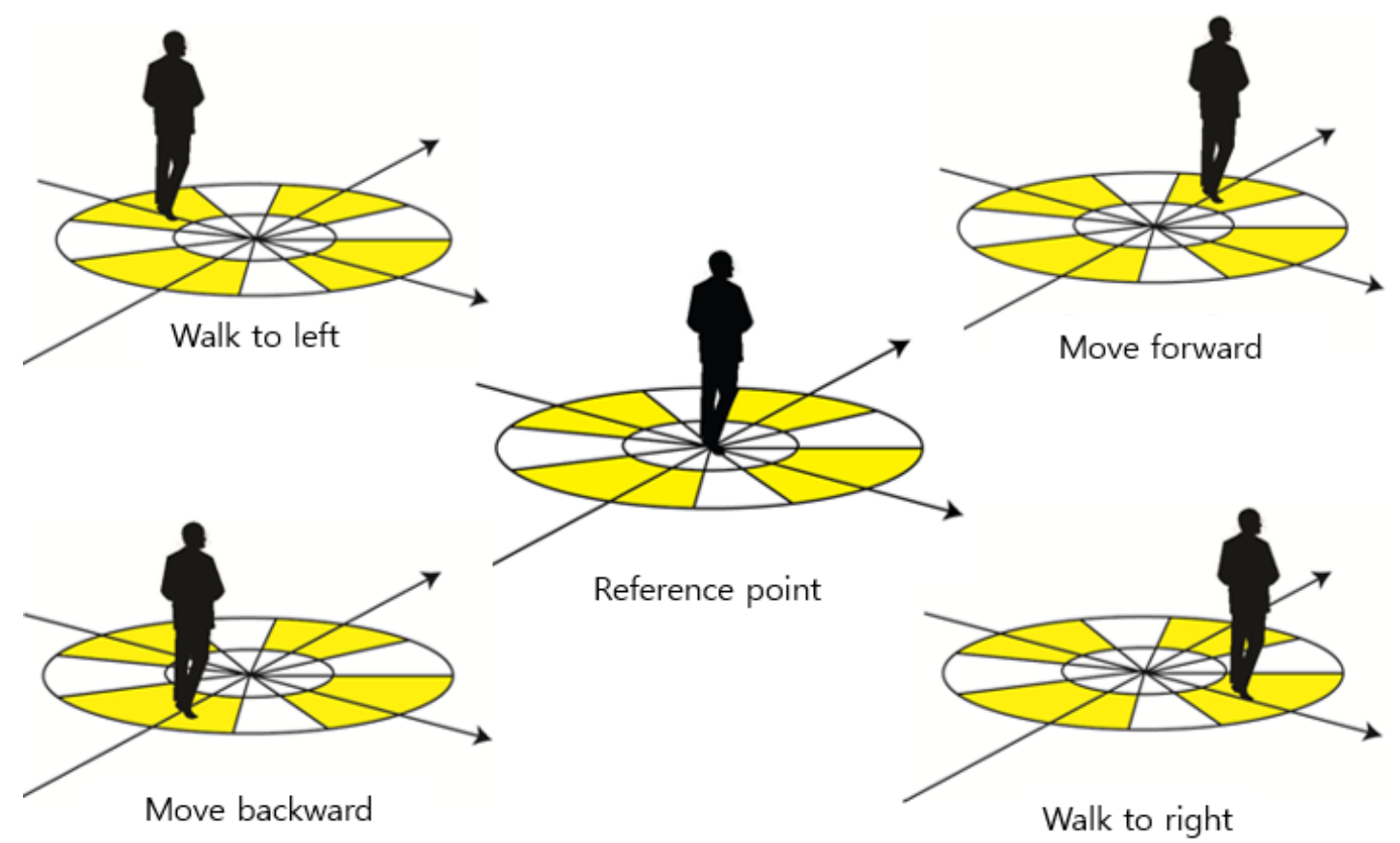

Fig. 2. Moving ranges of natural movement of navigation 
Table 3. Examples of Movement stimuli definition

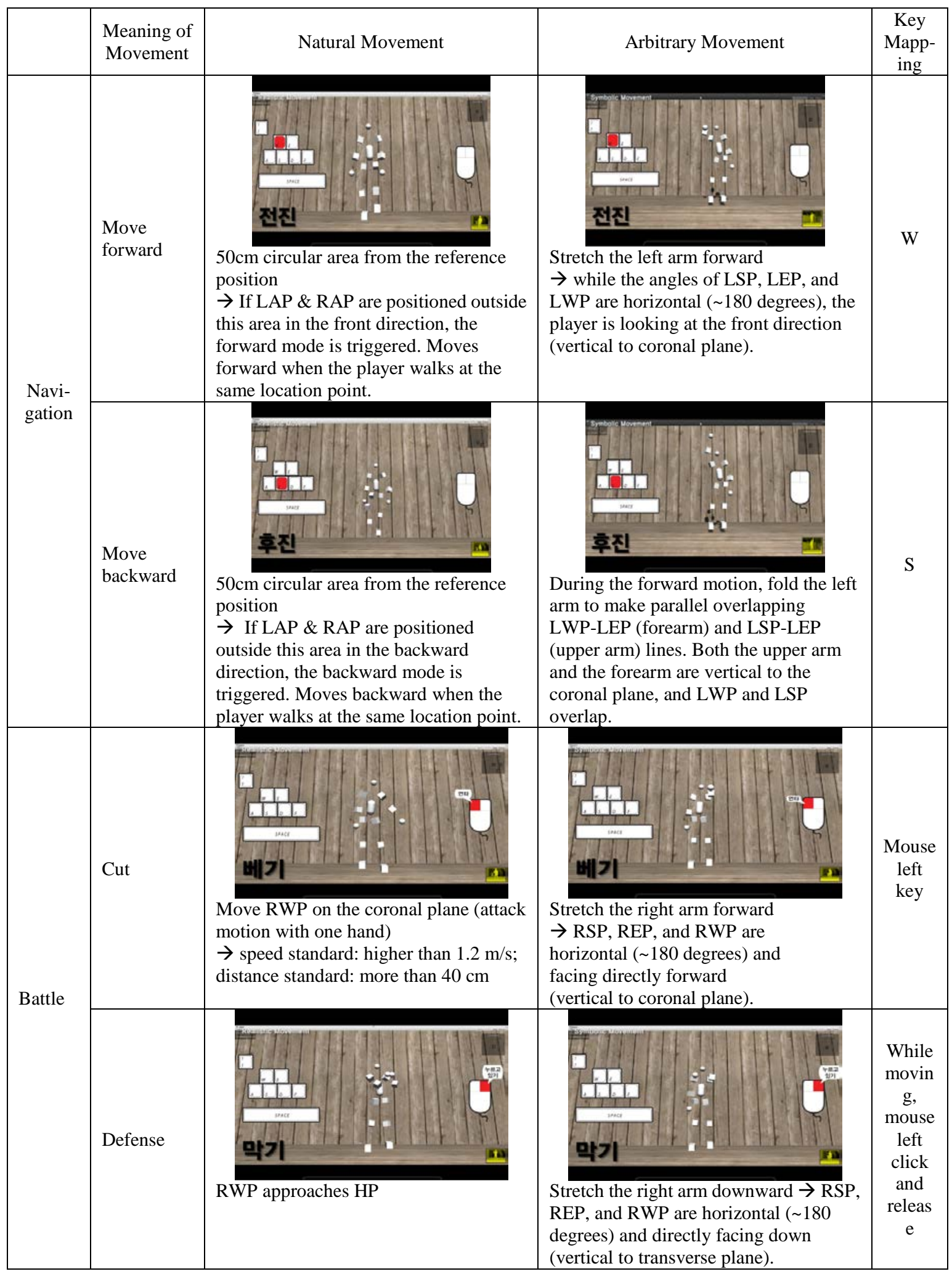




\subsection{Model for analysis}

Proposed herein is a model for the relationship between motion mapping and gaming performance. The natural characteristic of motion, whether natural or arbitrary, affects the players' game presence and enjoyment, and the players' recognition of a motion's reality can affect their game satisfaction and preference (Fig. 3). Statistics verification methods such as t-test and standard multiple regression were performed using SPSS 17.0.

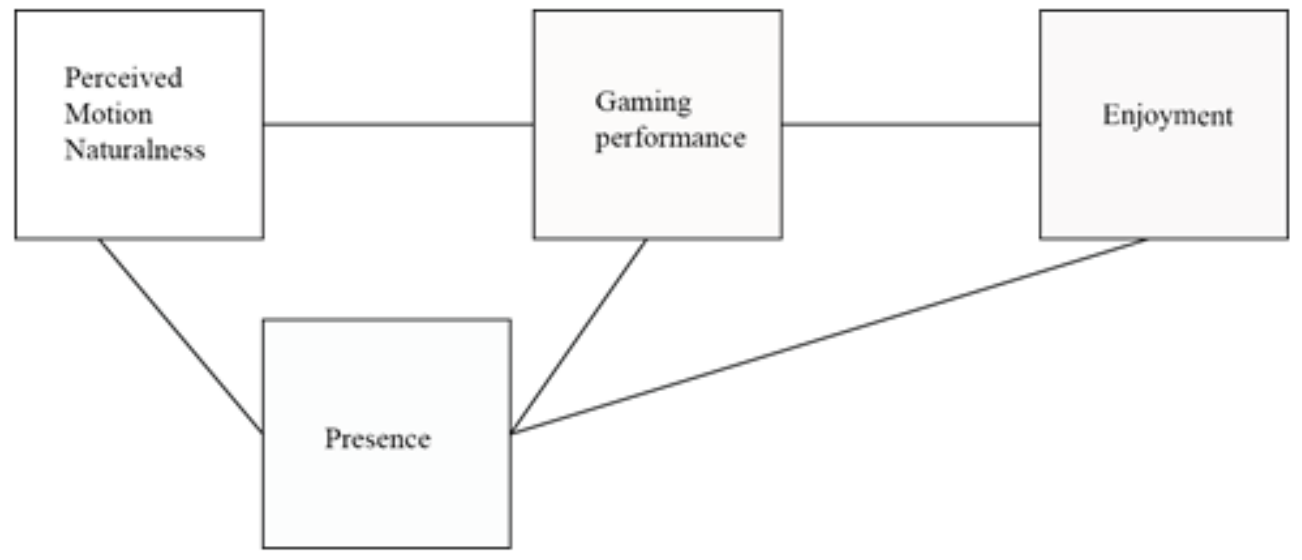

Fig. 3. Model for motion and game experience analysis

\section{Experimental Results}

In our approach, the mode of motion mapping positively predicts perceived motion naturalness because the meaning of naturalness differs according to individual thought. Nevertheless, the difference of perceived motion naturalness was significant in an independent sample t-test $(\mathrm{t}(78)=4.49, \mathrm{p}<0.01)$. The natural motion mapping condition was perceived as significantly more natural $(\mathrm{M}=4.87, \mathrm{SD}=0.95)$ than the arbitrary motion mapping condition $(\mathrm{M}=3.89, \mathrm{SD}=0.98)$.

The relationships between the rest of the factors were tested by a standard multiple regression approach with a two-block design. For investigating whether perceived motion naturalness positively predicts presence, the first block contained sex, age, skill level, prior motion-based game use, and game-play frequency as covariates. The second block added perceived motion naturalness to block 1 . All blocks accounted for a significant portion of the variance in presence, but compared to block 1 , block 2 (in which perceived motion naturalness was added) increased the variance accounted for $\left(\mathrm{R}^{2}=0.06, \mathrm{~F}(5,77)=5.78, \mathrm{R}^{2} \Delta=0.3, \mathrm{~F}(6\right.$, $77)=21.69, \mathrm{p}<0.01)$. The regression coefficient for perceived motion naturalness was significant $(\beta=0.62, \mathrm{t}(77)=6.0, \mathrm{p}<0.01)$. These results imply that players felt a higher level of presence as their perceived naturalness of motion increased.

Next, we tested whether presence positively predicts enjoyment. The method used for testing was the same as for the prior regression analysis. The first block contained all variables except presence, which was added in the second block. Block 1 accounted for a significant portion of the variance in presence $\left(\mathrm{R}^{2}=0.91, \mathrm{~F}(6,77)=4.86, \mathrm{p}<0.01\right)$. However, block 2 that was added presence to the model decreased variance accounted for $\left(\mathrm{R}^{2}=0.79, \mathrm{~F}(7,77)=3.21\right.$, $\mathrm{p}<0.05$ ). Thus, the portion of presence that influenced enjoyment was not significant, contrary to expectations. 
Finally, we examined whether gaming performance would positively predict enjoyment. This hypothesis was tested by regressing the variables from the two prior tests on game enjoyment, in two blocks. All the variables were contained in block 1, except for gaming performance. Block 1 accounted for a significant portion of the variance in enjoyment $\left(\mathrm{R}^{2}=\right.$ $0.59, \mathrm{~F}(7,77)=38.262, \mathrm{p}<0.01)$. Block 2 was also significant, but gaming performance was not the only significant predictor in the model $(\beta=0.13, \mathrm{t}(77)=60.168$, n.s. $)$.

As we expected, natural motion mapping contributed to players' perceived motion naturalness, presence, and gaming performance. In addition, these factors were positively related with game enjoyment. However, presence and gaming performance were not significant predictors in the model. Though these variables were not directly interactional, as we have suggested with the above model, indirect interaction could occur. Therefore, revising the model, we performed a path analysis.

A direct path from perceived motion naturalness to enjoyment, along with the result of the regression analysis, was included in the model. Then, presence and gaming performance were contained in the model. We used the least squares method to estimate the sizes of the model parameters and tested the overall model fit. Parameter size was estimated through regressing each endogenous variable onto its causal antecedent, and model fit was tested by comparing estimated parameter sizes to the reproduced correlations. Results for the model are shown in Fig. 4. The overall model fit was significant $\left(x^{2}=26.60, p=0.000\right)$.

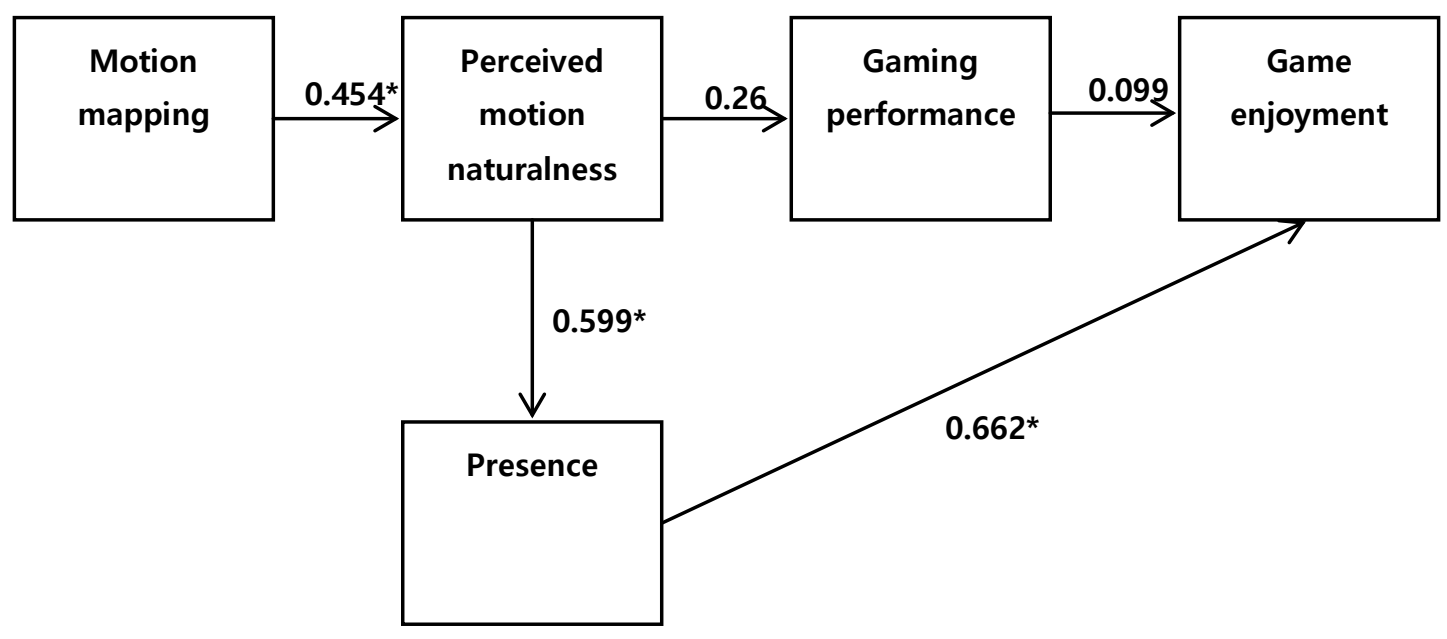

Fig. 4. Results for the path analysis of model $\left(*=p<0.01, x^{2}=26.60\right)$

Except for the path of perceived motion naturalness and gaming performance to game enjoyment, the rest of paths were significant. This implies that natural motion mapping influenced players' naturalness perception and that the perceived naturalness affected the level of presence. The level of presence ultimately influenced game enjoyment. However, the level of presence had no relevance to gaming performance, and perceived motion naturalness had little to do with gaming performance, as well. 


\section{Discussion}

\subsection{The remediation strategy of full-body motion game}

Games remediate previous media like novels or films. The game medium is categorized as taking the position of utilizing a non-mediating strategy more than any other type of media [11]. Due to the game medium's non-mediating strategy, game users cannot clearly distinguish between the medium world and the real world. This is referred to as new-media transparency. The gameplay method through player control improves the interactivity and maximizes the non-mediating characteristic, which no other media has achieved to date. Therefore, the research on the game medium and user can be divided into two categories: the re-mediating strategy focused on the media, and user experience and technology utilization. The media's re-mediating strategy can then be divided into transparency and hypertextuality. Interface design without an interface increases the transparency and can therefore maximize the users' non-mediative experience. From the viewpoint of the media, games based on full-body motion can be said to be the products of the most recent and innovative remodeling of the re-mediating method. The motion-based interface can provide the users with mediating experience and at the same time increases their game presence to improve their game immersion. As the sensory and motor embodiment levels are higher, the game player's game presence and enjoyment increase, leading to gameplay continuity [9]. Non-mediation, which the game medium is chasing, is being realized by the motion-based interface, and can enable the gameplay experience to reach the same level as actual experience.

Therefore, the player's embodiment type becomes more important than the player's embodiment level. The focus should be on player-focused motion reflection rather than on the game-focused command system. For mimetic game contents such as sports or dance, the player's motions and the game character's movement identification are the goals. This is why such games have been focused on the motion-based interface. It has been understood that other materials are not fit to go after motion identification. This paper emphasizes player-game character motion identification for the first-person RPG genre as a strategy to strengthen the gaming experience. Through this, it was checked if the player's gaming experience can be extended to real-life experience. For motions like looking backward or navigation, there were motion identification limitations because the character could not walk or look back, as in actual movement. The motion identification level was higher, however, in the battles, such as fighting one's enemies. As the game screen is placed in a fixed position, and as the screen does not move according to the player's view, complete motion identification is unavailable unless the player wears an HMD (head-mounted display) and the game is provided in a complete VR (virtual reality) environment. Despite these limitations, motion-based games extend the gaming experience to daily life experience, and therefore extend the game medium's non-mediating characteristic.

\subsection{Presence and enjoyment of full-body motion based game}

The mediating method of a new medium expands and remodels the previous media's way of mediating. For motion-based games, the game does not stay on a two-dimensional screen because the motion interface enables the players to identify the gaming experience as real-life experience. In this sense, the players' game presence and enjoyment develop into polyaesthetics [11] and can have the status of a motion-based interface. Outside the interface radius designed exclusively for games, the players can use daily motions in games. This enables the players to play the role of a gamer in terms of control and involvement, rather than the role of a mediator. Natural motions based on the players' mental model can increase the players' game performance, and through this, the players' game enjoyment and satisfaction 
can be maximized. This is because the sense of game experience that was previously based on the audiovisual senses is now being shifted to the sense of embodiment in terms of the motions. Multi-sensory aesthetics has not been discussed in the previous researches on the game interface. Such multi-sensory aesthetics can be acquired according to the players' motion type rather than motion level.

\section{Conclusion}

We have tried to explain the importance of natural motions in motion-based games for maximum game enjoyment. Natural motion mapping related to realistic motions in everyday life, but in motion-based games, it is difficult to reflect real-life motions. Because play areas are restricted, walking has to be represented by stepping in place, for example. However, through the experiment, we discovered that natural motion mapping influences players' perceptions of motion naturalness. When players could use natural motions, they felt those motions were realistic, and they felt like protagonists in the game, not merely game character controllers. This intimacy predicted the level of presence; thus, natural mapping affected players' presence. This means that the interface of realistic movements are changing the hypermediacy which is unique remediation of game media to immediacy.

However, the relationship between presence and gaming performance was not statistically significant. Prior research has shown that $90 \%$ of the variance in performance is explained by factors other than presence [6], and we confirmed that presence has nothing to do with gaming performance. In the end, the expression of natural motion is for greater game enjoyment, not for better performance.

Future research is planned to establish ideal natural motion mapping, to define the naturalness more specifically, and to develop ways of obtaining a sense of naturalness through players' motion design.

\section{References}

[1] M. G. Silva, D. A. Bowman., "Body-based interaction for desktop games,” in Proc. of CHI EA '09 27th international conference extended abstracts on Human factors in computing systems, 2009. Article (CrossRef Link)

[2] J. LaViola, "Bringing VR and Spatial 3D Interaction to the Masses through Video Games," IEEE, Computer Graphics and Application, IEEE, pp.10-15, 2008. Article (CrossRef Link)

[3] N. Bianchi-Berthouze,W.W. Kim, D. Pate, "Does body movement engage you more in digital game play? And why?,” Affective computing and intelligent interaction, 4738, pp. 102-113, 2007. Article (CrossRef Link)

[4] M. Pasch, N. Bianchi-Berthouze, B. Dijk, A. Nijholt, "Movement-based sports video games: Investigating motivation and gaming experience,” Entertainment Computing vol.1, pp.49-61, 2009. Article (CrossRef Link)

[5] G. Riva, "Is Presence a Technology Issue? Some insights from cognitive science,” Virtual Reality, vol.13, pp.159-167, 2009. Article (CrossRef Link)

[6] R. McGloin, K.M. Farrar, M. Krcmar, "The Impact of Controller Naturalness on Spatial Presence, Realism in a Tennis Simulation Video game,” Presence, vol.20, no.4, pp.309-324, 2011. Article (CrossRef Link)

[7] R. Tamborini, N. Bowman, "Presence in video games," Bracken CC. Skalski PD (eds) Immersed on Media: Telepresence in Everyday Life, Routledge, New York, pp.87-109, 2010.

[8] O.T. Kim, "The impact of video game's controller realism on natural mapping, spatial presence, arousal and emotions: using first-person shooting video games," Korean Journal of Journalism \& Communication Studies, vol. 54, no.4, pp.227-254, 2010. 
[9] S.Y. Kim, N. Prestopnik, F. A. Biocca, "Body in the interactive game : How interface embodiment affects physical activity and health behavior change,” Computers in Human Behavior, 36, pp.376-384, 2014. Article (CrossRef Link)

[10] P. Bayliss, "Being in the game-world: characters, avatars, and players," in Proc. of IE'07: Proceedings of the $4^{\text {th }}$ Australasian conference on Interactive entertainment, pp.1-6, 2007. Article (CrossRef Link)

[11] J.D. Bolter and R. Grusin, Remediation, Understanding New Media, The MIT Press, London, 1999.

[12] R. Farrow and I. Iacovides, “Gaming and the limits of digital embodiment," Philosophy and Technology, 27, 2014, pp.221-233. Article (CrossRef Link)

[13] M. Csikszentmihalyi, "Flow: The psychology of optimal experience,” Harper and Row, New York, 1990.

[14] L. Ermi and F. Mäyrä, "Fundamental components of the gameplay experience : analyzing immersion,” in Proc. of Proceedings of DiGRA 2005 conference, 2005. Article (CrossRef Link)

[15] J. H. Murray, "Hamlet on the Holodeck: The Future of Narrative in Cyberspace,” Free Press, New York, 1997. Article (CrossRef Link)

[16] E. Brown and P. Cairns, “A grounded investigation of Game Immersion,” CHI'04 Extended Abstracts on Human Factors and Computing systems, ACM Press, pp.1297-1300, 2004. Article (CrossRef Link)

[17] M. Slater and A. Steed, “A Virtual Presence Counter,” Presence, 9(5), pp.413-434, 2000. Article (CrossRef Link)

[18] R. Tan, and I. W. Davis, "Differential video coding of face and gesture events in presentation videos,” Computer Vision and Image Understanding, 96 (2), pp. 200-215, 2004. Article (CrossRef Link)

[19] B. Witmer and M.J. Singer, "Measuring presence in virtual environments : a presence questionnaire,” Presence, 7(3), pp 225-240, 1998. Article (CrossRef Link)

[20] C. Jennett, A. L. Cox, P. Cairns, S. Dhoparee, A. Epps, T. Tijs, A. Walton, "Measuring and defining the experience of immersion in games," International Journal of Human-Computer Studies, 66(2) pp.614-661, 2008. Article (CrossRef Link)

[21] J. D. Bolter, M. Engberg and B. Maclntyre, "Media, Studies, Mobile Augmented Reality, and Interaction Design,” Interactions, pp. 36-45, 2013. Article (CrossRef Link)

[22] T. Woo, "Video game culture and interactivity," Ph.D. Thesis. The university of Dundee, Scotland, United Kingdom, 2009.

[23] Z. Li, J. Tang, "Weakly Supervised Deep Metric Learning for Community-Contributed Image Retrieval,” IEEE TRANSACTION ON MULTIMEDIA, VOL.17, NO.11, pp.1989-1999, 2015. $\underline{\text { Article (CrossRef Link) }}$ 


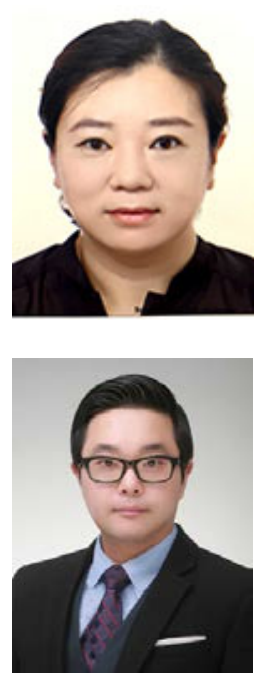

Ji Young Lee received the Ph.D degree in Cultural Technology from Korea Advanced Institute of Science and Technology DaeJeon-Si Korea in 2017. She did her master of Journalism in Ewha womans University Seoul Korea in 2003. Her research interests includes game design and players' experience design.

Tack Woo received the Ph.D degree in Electronic Imaging from University of Dundee Scotland United Kingdom in 2008. He is the chief professor in Digital Contents Faculty in Kyunghee University Suwon Korea. His research interests includes serious games, game interactivity and game development technology. 